\title{
Experimental realization of magnetic energy concentration and transmission at a distance by metamaterials
}

Jordi Prat-Camps, Carles Navau, and Alvaro Sanchez

Citation: Appl. Phys. Lett. 105, 234101 (2014);

View online: https://doi.org/10.1063/1.4903867

View Table of Contents: http://aip.scitation.org/toc/apl/105/23

Published by the American Institute of Physics

\section{Articles you may be interested in}

Experimental investigation of compact metamaterial for high efficiency mid-range wireless power transfer applications

Journal of Applied Physics 116, 043914 (2014); 10.1063/1.4891715

Experiments on wireless power transfer with metamaterials

Applied Physics Letters 98, 254101 (2011); 10.1063/1.3601927

Experiments on adjustable magnetic metamaterials applied in megahertz wireless power transmission AIP Advances 5, 017142 (2015); 10.1063/1.4907043

Magnetic superlens-enhanced inductive coupling for wireless power transfer Journal of Applied Physics 111, 064902 (2012); 10.1063/1.3692757

Extended explanation of transformation optics for metamaterial-modified wireless power transfer systems Journal of Applied Physics 119, 115105 (2016); 10.1063/1.4943774

Electromagnetic time reversal focusing of near field waves in metamaterials

Applied Physics Letters 109, 263901 (2016); 10.1063/1.4973210

\section{Scilight}

Sharp, quick summaries illuminating the latest physics research

\section{Sign up for FREE!}

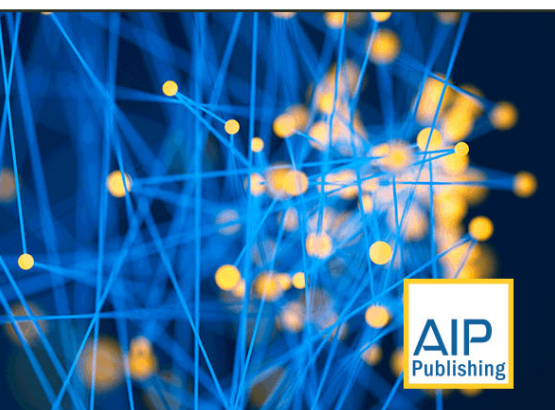




\title{
Experimental realization of magnetic energy concentration and transmission at a distance by metamaterials
}

\author{
Jordi Prat-Camps, Carles Navau, and Alvaro Sanchez \\ Departament de Física, Universitat Autònoma de Barcelona, 08193 Bellaterra, Barcelona, Catalonia, Spain
}

(Received 16 October 2014; accepted 28 November 2014; published online 9 December 2014)

\begin{abstract}
Concentrating magnetic energy in a desired volume is an important requirement for many technologies. Here, we experimentally realize a superconductor-ferromagnetic metamaterial that allows to concentrate the magnetostatic energy in its interior and in other situations to amplify the energy in its exterior. We show that surrounding two distant current loops with two such metamaterials enhance the magnetostatic coupling between them. We also demonstrate that a ferromagnetic-only metamaterial, without superconducting parts, achieves these properties with only a slight decrease in performance. Results may be applied to increase the sensitivity of magnetic sensors or for enhancing wireless power transmission, where efficiency depends critically on the magnetic coupling strength between source and receiver. (C) 2014 AIP Publishing LLC.

[http://dx.doi.org/10.1063/1.4903867]
\end{abstract}

Metamaterials have opened new possibilities to control electromagnetic fields, ${ }^{1-5}$ in particular, for concentrating energy at different scales and wavelengths. Examples range from electromagnetic energy concentration by plasmonics at the nanoscale ${ }^{6,7}$ to thermal energy concentration at larger scale. ${ }^{8,9}$ Metamaterials have also provided new ways of controlling static magnetic fields, often by applying the ideas of transformation optics to the static dc case. ${ }^{10-14}$ For example, magnetic cloaks have been proposed and experimentally realized using superconductors combined with magnetic materials. ${ }^{15-18}$ Similar ideas have been applied to the realization of dc electrical cloaks and other devices. ${ }^{19,20}$

We theoretically introduced in Ref. 21, based on transformation optics, a cylindrical metamaterial shell with extreme radial and angular permeability components $\mu_{\rho} \rightarrow \infty$ and $\mu_{\theta} \rightarrow 0$, that concentrates an externally applied static magnetic field in its interior. The shell also expels the field of an interior source to the exterior, increasing its magnitude. Both concentration and expulsion factors increase as the ratio of outer to inner shell radii, which can be tuned to achieve large values. The combination of these concentration and expulsion properties can be used to achieve field concentration at points distant from the source through free space. ${ }^{21}$ The ideal concentrating shell required extreme magnetic properties, not directly found in natural materials, and its length was assumed infinite. Magnetic metamaterials made of alternated superconductor (SC) and ferromagnetic (FM) pieces could represent a good approximation to the required anisotropic permeabilities. $^{21,22}$ Other studies have proposed to concentrate static magnetic fields using related ideas with other geometries, ${ }^{23}$ and also in arrangements involving magnets. ${ }^{24}$

In this work, we develop and construct actual metamaterial shells to experimentally confirm the properties of magnetic concentration, expulsion, and concentration at a distance. We do not only validate the theoretical ideas of Ref. 21 but extend them by demonstrating another important property, i.e., metamaterial shells composed of only FMs separated with air gaps (without the use of SCs and their associated cryogenics) show a good performance as well.
We build a metamaterial cylindrical shell made of 36 rectangular prisms of alternated SC and FM materials (see supplemental material Sec. A and Fig. S1 for details ${ }^{25}$ ). The FM and SC pieces are made of commercial highpermeability metallic alloy (mu-metal) and coated conductor, respectively. The pieces are radially distributed and fixed in a non-magnetic 3D-printed support (Figs. S1(b) and S1(c)).

We first experimentally study the magnetic concentration properties of our metamaterial shell. Two Helmholtz coils created a uniform external field $\left(B_{\mathbf{a}}\right)$ perpendicular to the shell axis. A Hall probe was used to measure the concentrated field $\left(B_{z}^{\mathrm{IN}}\right)$ in the central point inside the shell (see a sketch in Fig. 1(a)). Measurements were first performed with the metamaterial submerged in liquid nitrogen, at $77 \mathrm{~K}$, below the critical temperature of the SC, $T_{c}$ ( $92 \mathrm{~K}$ for the YBCO superconductor we use). Measurements below $T_{c}$ showed that field was increased by a factor 2.70 (green symbols and dashed fitting line in Fig. 1(b)). This number is in excellent agreement with the 3D finite-element numerical simulation of this system (green solid line and Fig. 1(d)), which considered the actual finite dimensions and assumed ideal magnetic permeabilities of the materials $\left(\mu_{\mathrm{FM}} \rightarrow \infty\right.$ and $\mu_{\mathrm{SC}} \rightarrow 0$; see supplementary material for simulation details). The magnetic-field enhancement factor at the center for the ideal infinitely long metamaterial should be 4 , corresponding to the ratio between outer and inner shell radii, ${ }^{21}$ the difference mainly owing to the finite size of the metamaterial and its discretized nature. Simulation and experiments also show that, despite the finite shell length, magnetic field achieves a very homogenous concentration. ${ }^{21}$

We next study if similar results can be produced with simplified versions of the metamaterial. In particular, it would be very useful to avoid the use of superconducting parts, which require cryogenic temperatures. We confirm this by measuring the concentration of magnetic field at room temperature, at which the SCs are above $T_{c}$ and thus deactivated (experiments show no magnetic signal from the SC above $T_{c}$; see Sec. B of supplemental material ${ }^{25}$ ). A 
(a)

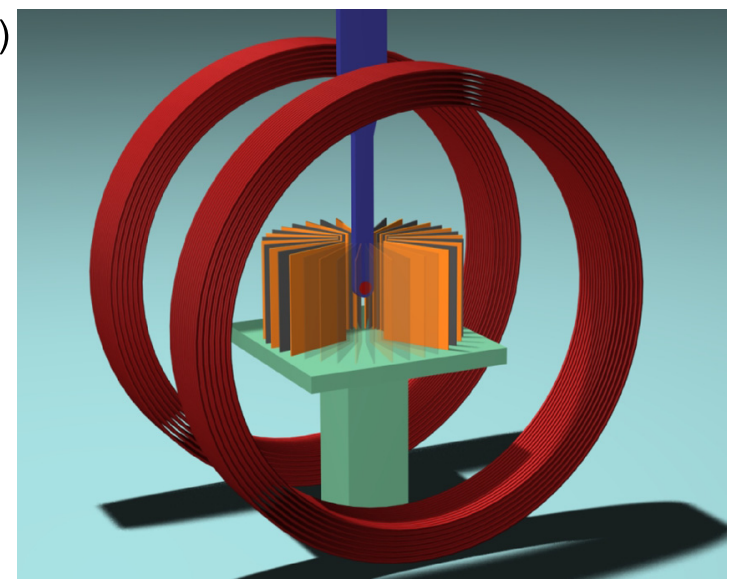

(b)

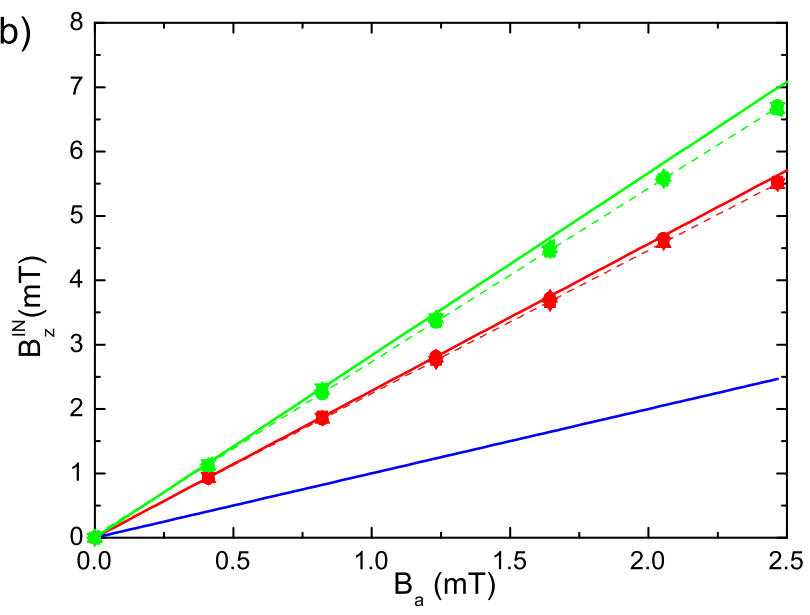

(c)

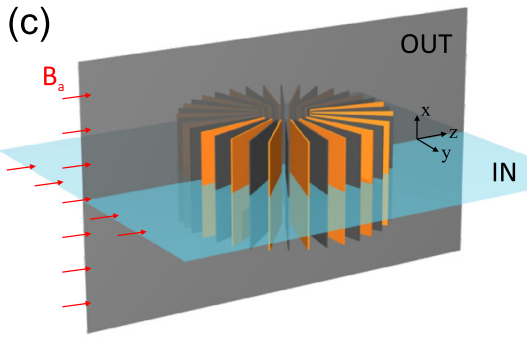

(d) IN

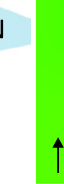

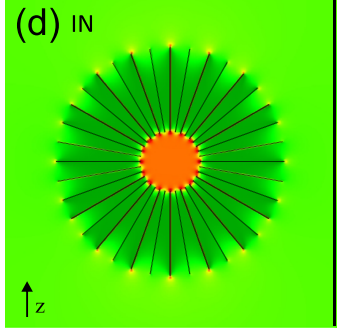
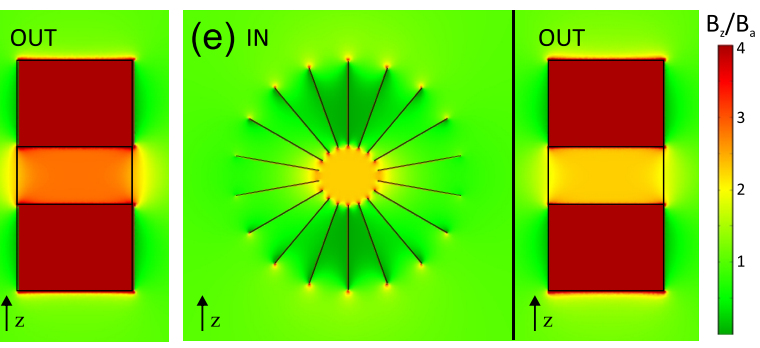

FIG. 1. Magnetic field concentration. (a) Sketch of the experimental setup. Two Helmholtz coils (red) with a radius of 70 mm were used to create a uniform field. The metamaterial shell was placed in the central region (SC pieces represented in orange and FM ones in dark gray) and the Hall probe (blue) measured the field in the central interior point (some pieces have been sketched translucent to show the precise measuring point). (b) The measured field inside the metamaterial shell is plotted as a function of the field applied by the coils $B_{a}$ (in symbols) and for two different working conditions; measurements for $T<T_{c}$ in green and for $T>T_{c}$ in red (horizontal and vertical error bars $\Delta B_{a}=0.04 \mathrm{mT}$ and $\Delta B^{\mathrm{IN}}=0.002 \mathrm{mT}$, respectively, have been omitted for clarity). The corresponding linear fits are presented in dashed lines (whose slopes are 2.70 and 2.23 for $T<T_{c}$ and $T>T_{c}$, respectively), together with the simulations results in solid lines (whose slopes are 2.83 and 2.28 for the $18 \mathrm{FM}+18 \mathrm{SC}$ and $18 \mathrm{FM}$ shells, respectively), showing a good agreement. The blue line shows the field created by the bare coils in the central point and is relevant to show the improvement achieved by the metamaterial shell in both working conditions. The calculated $B_{z}$ field component is plotted in the planes IN and OUT, (c), for the case of $18 \mathrm{SC}+18 \mathrm{FM}$ (i.e., $T<T_{c}$ ), (d), and for the case of only 18 FM $\left(T>T_{c}\right)$, (e).

concentrating factor of 2.23 (red symbols and dashed fitting line in Fig. 1(b)) was still achieved under these conditions. The corresponding simulation with only 18 FM pieces (red solid line and Fig. 1(e)) perfectly matches these measurements. Simulations also illustrate the role played by the two different materials. The SC layers in the SC-FM metamaterial (Fig. 1(d)) prevent angular components of field in the shell volume, so that field is basically guided radially to the central hole by the FM pieces. In the FM-only metamaterial shell (Fig. 1(e)), this role played by the SC parts is now partially taken up by the air gaps.

We next experimentally study the expelling properties of our metamaterial shell by measuring the field at its exterior when a dipolar-like magnetic field source is in the shell interior. A small coil was placed at the center of the shell with its axis on the $z$ direction (see Fig. 2(a)) and fed with a constant current. The field $B_{z}$ was measured outside the shell as a function of the distance $z$. Measurements below $T_{c}$ (green symbols in Fig. 2(b)) show the expelled field increased by a factor of around 2.5 with respect to the bare coil (blue symbols). Above $T_{c}$, the shell also increased the field in all exterior points by a factor of around 2.0, confirming its good behavior even without SCs. These measurements agree well with the corresponding 3D finite-element simulations performed assuming ideal materials (solid lines in Fig. 2(b) and Figs. 2(c)-2(e)). Plots of the field at median planes show how the metamaterial shell expels most of the field from its interior to the exterior (Fig. 2(d)). When using solely FM pieces (Fig. 2(e)) the field expulsion is reduced.

The combination of the expulsion and concentration properties can be used to transfer magnetic energy at a distance, as theoretically anticipated in Ref. 21 . To experimentally demonstrate this property, we use the metamaterial shell with a coil inside as a source and a second metamaterial shell consisting of $18 \mathrm{FM}$ pieces (made of the same mumetal foil) with a rectangular shape similar to that of the first shell as a receiver (see Figs. 3(a) and 3(b)). We separate both shells a distance of $70 \mathrm{~mm}$ from center to center, so there is an air gap of $10 \mathrm{~mm}$ between them. Measurements are shown in Fig. 3(c), for two cases. When $T<T_{c}$ (upper plot), the shell with the source behaves like a full SC-FM metamaterial and, even though the receiver shell is a FM-only metamaterial, the measured transferred field is increased by an average factor of around 7.5 with respect to the field of the naked coil. When $T>T_{c}$ (lower plot), the average factor is still around 6.0. In both cases, it is seen that not only the field is magnified in the inner region of the receiver shell but also its gradient, as predicted in Ref. 21.

These experiments are a proof-of-principle of the theory for magnetic energy transmission with metamaterials; the particular obtained values can be further optimized. The field-increase factor of 7.5 or 6.0 and also the air gap distance, can be both turned into larger values by changing the geometry, like increasing the shells radii ratio. ${ }^{21} \mathrm{We}$ 

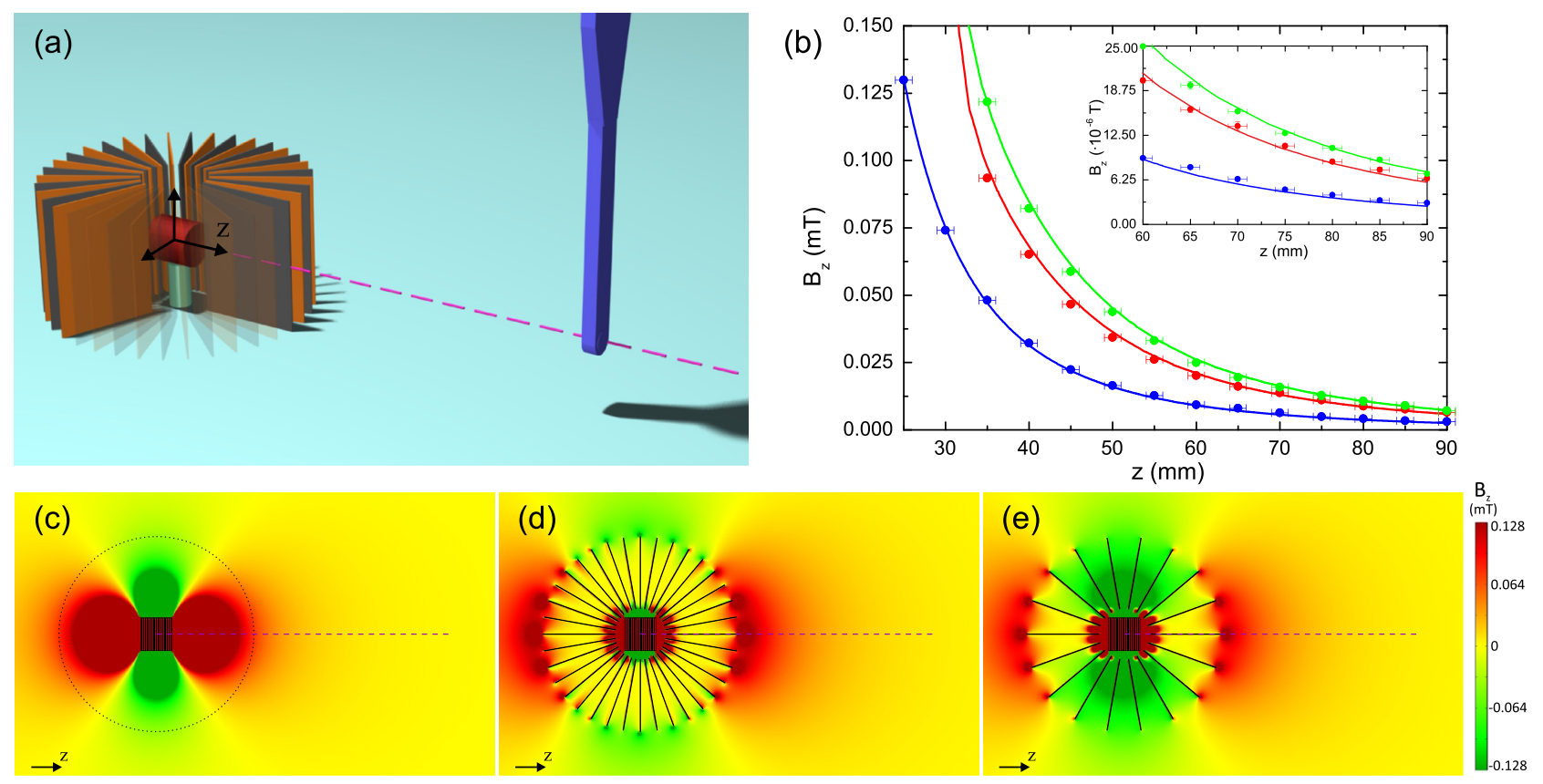

FIG. 2. Magnetic field expulsion. (a) Sketch of the experimental setup used to measure the expulsion capabilities of the shell. A small coil (red) was placed inside the metamaterial shell and was fed with a DC current. The $B_{z}$ field component was measured outside the shell by the Hall probe along the centered line (pink) as a function of the distance to the center $z$. (b) Measurement results are plotted in symbols; blue symbols correspond to the field of the isolated coil, red symbols are measurements outside the shell at $T>T_{c}$, and green ones when the measurements were performed at $T<T_{c}$. Solid lines show the results of the corresponding 3D simulations, in full agreement with the measurements. (c)-(e) Plots of the $B_{z}$ field component in the median plane of these simulations for the case of the coil alone, (c), $18 \mathrm{FM}+18 \mathrm{SC}\left(T<T_{c}\right)$, (d), and $18 \mathrm{FM}\left(T>T_{c}\right)$, (e).

(a)

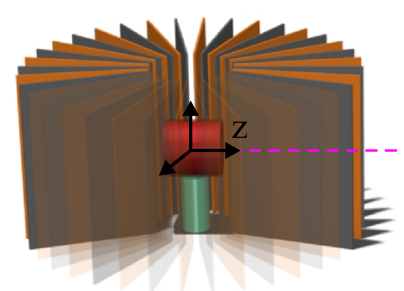

(b)

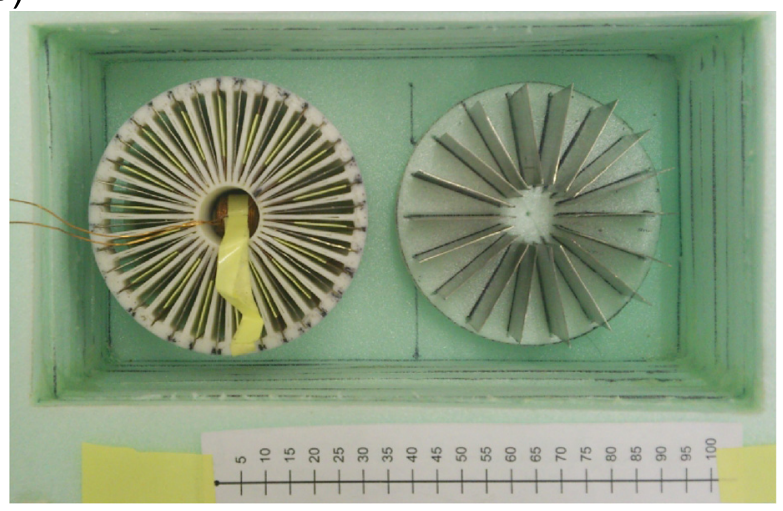

(c)
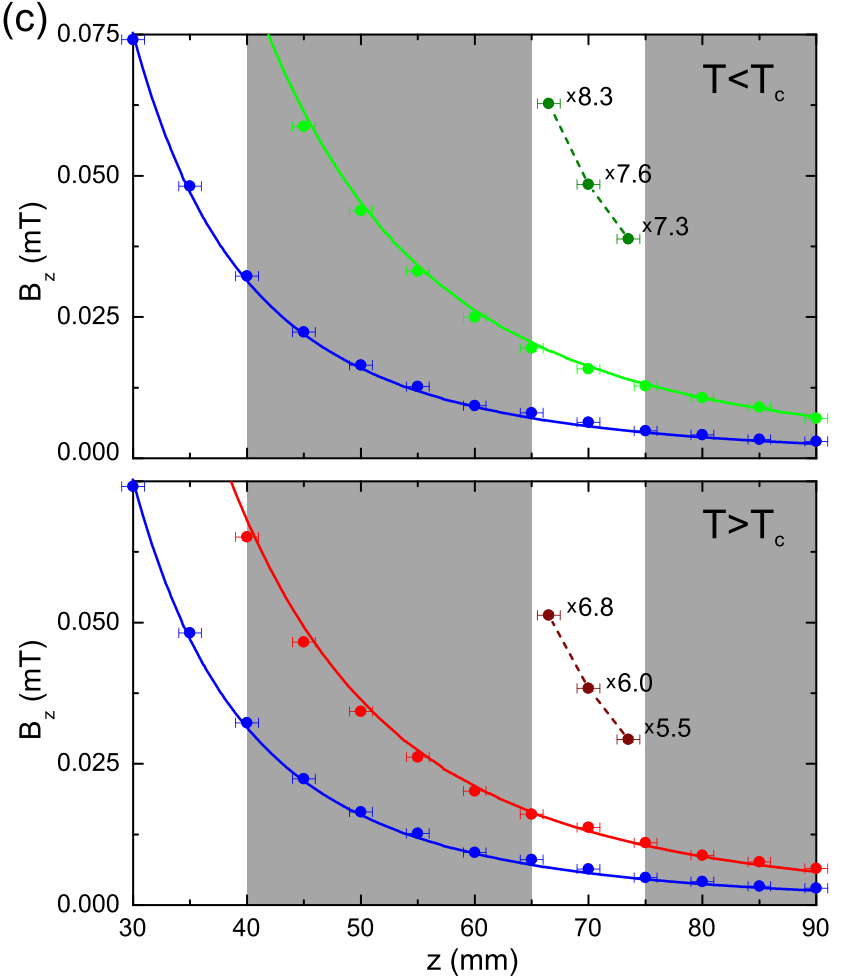

FIG. 3. Magnetic field concentration at a distance. (a) Sketch of the experimental setup. The coil (red) was placed inside the metamaterial shell. A second metamaterial shell with a similar size made of only $18 \mathrm{FM}$ pieces was placed at a distance of $70 \mathrm{~mm}$ from the center of the first shell. The field was measured by the Hall probe inside the second shell aligned with the coil. (b) Picture of the real experimental setup, with the coil placed inside the original metamaterial shell (left), and the FM-only metamaterial shell (right). (c) Measurement results (symbols) for the two working conditions; $T<T_{c}$ (upper plot) and $T>T_{c}$ (lower plot). In the upper plot, blue symbols and line correspond to measurements and simulation of the coil alone, and light green symbols and line correspond to measurements and simulation of the field expelled by the metamaterial shell at $T<T_{c}$. (These results were already presented in the previous figure and are only included for comparison.) The dark green symbols correspond to the field measured inside the second shell when both shells are present (dashed line is a guide for the eye). The numbers next to the symbols indicate the enhancement factor of the measured field with respect to the field created by the bare coil at the same positions. In the lower plot, blue symbols and line correspond to the case of the bare coil, red symbols and line represent the expelling measurements and simulation at $T>T_{c}$, and dark red symbols correspond to the field measured inside the second shell when the two shells are present (dashed line is a guide for the eye). The enhancement factors are also shown. 
demonstrate this by constructing a new shell with a larger radii ratio $\left(R_{2} / R_{1}=5\right)$ and measuring its expelling-field properties. Measurements of the field expelled at $T<T_{c}$ when a small coil is placed inside it are shown in Fig. S3. The ratio of the field measured at a given point resulting from the new shell with large radii ratio with respect to the field created by the coil alone at the same point is around 3.5 (see supplemental material Sec. C for details ${ }^{25}$ ), whereas for the shell with smaller radii this ratio was around 2.5. These experiments confirm that larger radii ratio of the concentrator results in larger field expulsions.

When a second coil is placed in the receiver shell, the enhanced field transmission is equivalent to increasing the magnetic coupling between both coils, which may have applications in wireless transmission of power. ${ }^{26,27}$ In wireless systems, including the recent non-radiative power transfer proposals, ${ }^{28}$ the mutual inductive coupling between the source and the receive resonators is a key parameter to increase the efficiency. As discussed in Ref. 29, because the distance between the source and receiver is so much smaller than the wavelength, the relevant field distribution is quasistatic and the inductive coupling relates predominantly to magnetic flux emanating from one coil captured by the second coil. This is precisely what we achieve with our metamaterial shells. Actually, magnetic metamaterials have been already proposed for increasing the magnetic coupling in wireless systems in the form of magnetic lenses. This strategy requires a negative permeability material, ${ }^{29-33}$ whereas our metamaterial is made of non-resonant materials with positive permeability.

Even though our ideas have been confirmed strictly in the static limit, there are good indications that for low frequencies (up to tenths of kilohertz at least) the materials will behave as required. Recently, a magnetic cloak made of similar FM and SC materials designed to operate at the dc regime was shown to maintain cloaking properties for lowfrequency applied magnetic fields. ${ }^{34}$ Preliminary experimental results indicate that wireless transmission of power between circuits at some frequencies of interest will be enhanced by using our metamaterial shells.

To sum up, we have experimentally demonstrated the unique properties that superconductor-ferromagnetic metamaterials can offer in terms of magnetic energy concentration, expulsion, and transmission through free space. Although the original theory was derived for infinite samples and assumed ideal materials, our results confirm that these properties can be achieved in finite geometries and using commercially available materials. Finite-element calculations precisely reproduce the measured data and thus can be used to obtain optimized designs. Even when the superconducting parts are removed, very good behaviour is obtained using only ferromagnetic metamaterials at room temperature. Also, modifications of the studied magnetic metamaterials can be explored as well for changing the magnetic field shape in the space around the shell in other desired ways. Possible application of the results to technology include, in particular, increasing the magnetic coupling between distant circuits, an essential factor for enhancing wireless power transmission.

We thank Fedor Gomory and Jan Souc for helpful discussions, Elena Bartolome and EUSS for support, and SuperPower for providing the coated conductors. We also thank Spanish project MAT2012-35370 for financial support. A.S. acknowledges a grant from the ICREA Academia, funded by the Generalitat de Catalunya. J.P.C. acknowledges a FPU grant form Spanish Government (AP2010-2556).

${ }^{1}$ T. J. Cui, D. R. Smith, and R. Liu, Metamaterials: Theory, Design and Applications (Springer, New York, 2010).

${ }^{2}$ N. I. Zheludev and Y. S. Kivshar, Nature Mater. 11, 917 (2012).

${ }^{3}$ J. B. Pendry, A. J. Holden, D. J. Robbins, and W. J. Stewart, IEEE Trans. Microwave Theory Tech. 47, 2075 (1999).

${ }^{4}$ J. B. Pendry, D. Schurig, and D. R. Smith, Science 312, 1780 (2006).

${ }^{5}$ H. Chen, C. T. Chan, and P. Sheng, Nature Mater. 9, 387 (2010).

${ }^{6}$ J. A. Schuller, E. S. Barnard, W. Cai, Y. C. Jun, J. S. White, and M. L. Brongersma, Nature Mater. 9, 193 (2010).

${ }^{7}$ A. Aubry, D. Y. Lei, A. I. Fernandez-Dominguez, Y. Sonnefraud, S. A. Maier, and J. B. Pendry, Nano Lett. 10, 2574 (2010).

${ }^{8}$ S. Narayana and Y. Sato, Phys. Rev. Lett. 108, 214303 (2012).

${ }^{9}$ T. Han, J. Zhao, T. Yuan, D. Lei, B. Li, and C.-W. Qiu, Energy Environ. Sci. 6, 3537-3541 (2013).

${ }^{10}$ B. Wood and J. B. Pendry, J. Phys. Condens. Matter 19, 076208 (2007).

${ }^{11}$ F. Magnus, B. Wood, J. Moore, K. Morrison, G. Perkins, J. Fyson, M. C. K. Wiltshire, D. Caplin, L. F. Cohen, and J. B. Pendry, Nature Mater. 7, 295 (2008).

${ }^{12}$ C. Navau, D.-X. Chen, A. Sanchez, and N. Del-Valle, Appl. Phys. Lett. 94, 242501 (2009).

${ }^{13}$ S. M. Anlage. J. Opt. 13, 024001 (2011).

${ }^{14}$ P. Jung, A. V. Ustinov, and S. M. Anlage, Supercond. Sci. Technol. 27, 073001 (2014).

${ }^{15}$ A. Sanchez, C. Navau, J. Prat-Camps, and D.-X. Chen, New J. Phys. 13, 093034 (2011).

${ }^{16}$ S. Narayana and Y. Sato, Adv. Mater. 24, 71 (2012).

${ }^{17}$ F. Gomory, M. Solovyov, J. Souc, C. Navau, J. Prat-Camps, and A. Sanchez, Science 335, 1466 (2012).

${ }^{18}$ R. Wang, Z. L. Mei, and T. J. Cui, Appl. Phys. Lett. 102, 213501 (2013).

${ }^{19}$ F. Yang, Z. L. Mei, T. Y. Jin, and T. J. Cui, Phys. Rev. Lett. 109, 053902 (2012).

${ }^{20}$ M. Liu, Z. L. Mei, X. Ma, and T. J. Cui, Appl. Phys. Lett. 101, 051905 (2012).

${ }^{21}$ C. Navau, J. Prat-Camps, and A. Sanchez, Phys. Rev. Lett. 109, 263903 (2012).

${ }^{22}$ J. Prat-Camps, A. Sanchez, and C. Navau, Supercond. Sci. Technol. 26, 074001 (2013).

${ }^{23}$ F. Sun and S. He, Prog. Electromagn. Res. 142, 579 (2013).

${ }^{24}$ R. Bjork, A. Smith, and C. R. H. Bahl, J. Appl. Phys. 114, 053912 (2013).

${ }^{25}$ See supplementary material at http://dx.doi.org/10.1063/1.4903867 for details on sample preparation and experimental methods, and for further experimental data.

${ }^{26}$ S. Stewart, Science 317, 55 (2007).

${ }^{27}$ S. Kim, J. S. Ho, and A. S. Y. Poon, Phys. Rev. Lett. 110, 203905 (2013).

${ }^{28}$ A. Kurs, A. Karalis, R. Moffatt, J. D. Joannopoulos, P. Fisher, and M. Soljacic, Science 317, 83 (2007).

${ }^{29}$ D. Huang, Y. Urzhumov, D. R. Smith, K. H. Teo, and J. Zhang, J. Appl. Phys. 111, 064902 (2012).

${ }^{30}$ J. Choi and C. Seo, Prog. Electromagn. Res. 106, 33 (2010).

${ }^{31}$ Y. Urzhumov and D. R. Smith, Phys. Rev. B 83, 205114 (2011).

${ }^{32}$ B. Wang, W. Yerazunis, and K. H. Teo, Proc. IEEE 101, 1359 (2013).

${ }^{33}$ G. Lipworth, J. Ensworth, K. Seetharam, D. Huang, J. S. Lee, P. Schmalenberg, T. Nomura, M. S. Reynolds, D. R. Smith, and Y. Urzhumov, Sci. Rep. 4, 3642 (2014).

${ }^{34}$ J. Souc, M. Solovyov, F. Gomory, J. Prat-Camps, C. Navau, and A. Sanchez, New J. Phys. 15, 053019 (2013). 